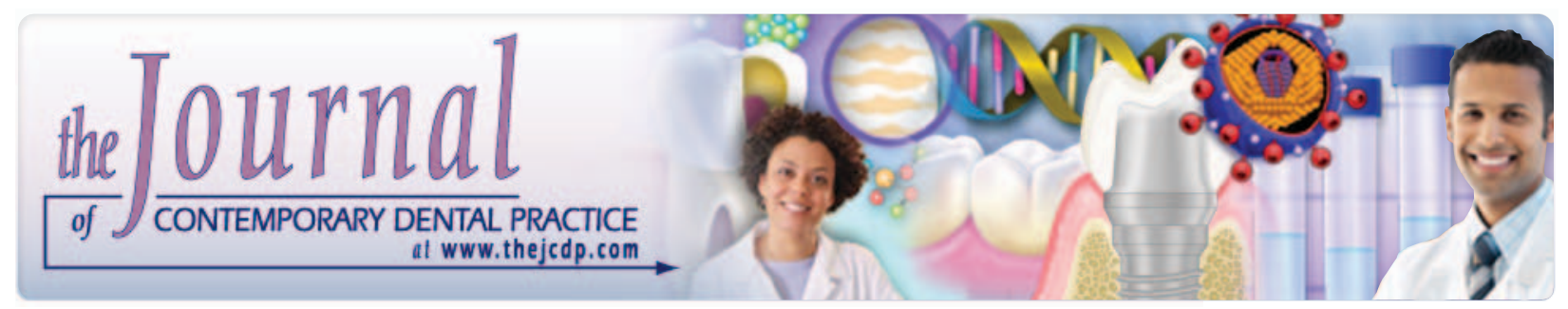

\title{
Occlusal Rehabilitation in a Partially Edentulous Patient with Lost Vertical Dimension Using Dental Implants: A Clinical Report
}

Azam Sadat Madani, DDS, MS; Amir Moeintaghavi, DDS, MS; Maryam Rezaeei, DDS,MS

\section{Abstract}

Aim: This clinical report describes the occlusal rehabilitation of a partially edentulous patient with lost vertical dimension.

Background: The patient did not want a removable partial denture. Implants, fixed restorations with occlusal plane correction, were used to restore the anterior and posterior support and treat the severely worn dentition. However, a partially edentulous patient may be unable to recover normal function, esthetics, comfort, or phonetics with a conventional removable dental prosthesis. The use of dental implants-supported fixed prosthesis offers a multitude of benefits over a tooth-soft tissue supported removable partial denture prosthesis.

Summary: This clinical report describes an organized approach to an occlusal reconstruction of a severely worn dentition, partially edentulous patient with fixed restorations, implants, occlusal plane correction, and recovery of the vertical dimension. The treatment offered the patient improved esthetics and the restoration of oral function.

Clinical Significance: The main indications for implant-supported restorations in a partially edentulous patient are the elimination of the freeend distal extension and the benefit from fixed restorations. Success with implant-supported prosthodontics needs the same or more attention to detail and careful treatment planning for conventional fixed prosthodontics; a team approach is recommended.

Keywords: Occlusal rehabilitation, implant, vertical dimension, case report.

Citation: Madami AS, Moeintaghavi A, Rezaeei M. Occlusal Rehabilitation in a Partially Edentulous Patient with Lost Vertical Dimension Using Dental Implants: A Clinical Report. J Contemp Dent Pract (internet]. 2010 December; 11(6):058-064. Available from http://www.thejcdp. $\mathrm{com} /$ journal/view/volume11-issue6-rezaeei

\section{Introduction}

Conventional prosthodontic treatment planning for partially edentulous patients has changed significantly since the introduction of dental implants.

The traditional treatment for a partially edentulous patient used to be the fabrication of a removable partial denture (RPD). Although an RPD has great benefits, removable soft tissue-borne partial dentures have one of the lowest patient acceptance rates in dentistry. ${ }^{1}$ It is estimated that approximately one-half the number of persons with a removable partial denture chew better without the prosthesis. ${ }^{1}$

Occlusion is difficult to establish and maintain with a completely soft tissue-supported prosthesis, especially in a patient with a worn dentition. ${ }^{\text {? }}$ 
The successful long-term use of osseointegrated implants to treat edentulous patients has been expanded to partially edentulous patients. ${ }^{-}$Dental implants stimulate the bone and help to maintain bone levels much like the presence of healthy natural teeth. Consequently, an implant-supported restoration improves oral comfort. ${ }^{1}$

Indications for implant placement in a partially edentulous patient include the following: (1) inability to wear a removable partial denture, (2) need for long-span fixed dental prosthesis with questionable prognosis, and (3) unfavorable number and location of potential tooth abutments. ${ }^{4}$

Implant treatment is also indicated for those patients with a compromised dentition who require complete oral rehabilitation. Successful restoration with implant-supported prostheses has even been reported in the treatment of patients with extreme occlusal wear. ${ }^{5.6}$ Implant success reported from major research institutions is quite high. ${ }^{4}$ However, attention to patient selection, diagnosis, and treatment planning is necessary to achieve this success.

This clinical report describes the occlusal rehabilitation of a partially edentulous patient for whom dental implants and fixed restorations were used to restore severely worn dentition. Restoration of the patient's lost vertical dimension of occlusion was necessary as part of the oral rehabilitation.

\section{Clinical Report}

\section{Diagnosis}

A 63-year-old man presented with the chief complaint of difficulty chewing and poor esthetics. Detailed medical and dental histories were obtained and indicated the patient was in good general medical health. However, the extraoral examination revealed that the patient had reduced face height due to the loss of vertical dimension of occlusion (VDO). The intraoral examination revealed the absence of several teeth, the severe loss of tooth structure from the lingual cusps of the maxillary teeth, severe wear of mandibular incisor teeth, and a Class II division 1 malocclusion (Figures 1, 2, and 3). The patient did not wear a removable partial denture. Cone- beam tomography (CT) scans, made by an oral radiologist, were used to identify implant sites and guide implant placement.

The occlusal plane was uneven, and the VDO was reduced secondary to loss of posterior support, as illustrated in Figures 2 and 3.

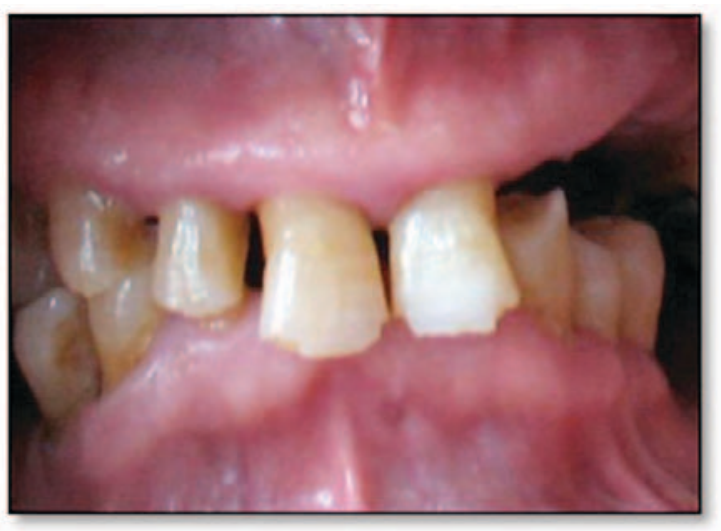

Figure 1. The pretreatment appearance of the patient (anterior view).

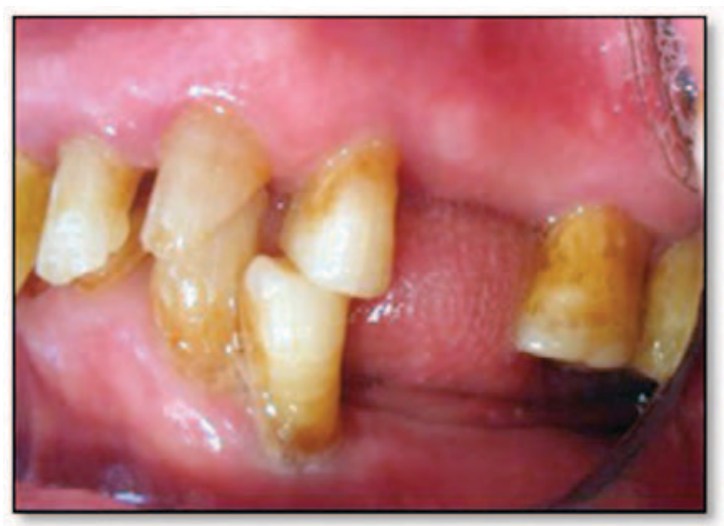

Figure 2. Pretreatment appearance (mirror view of patient's right side).

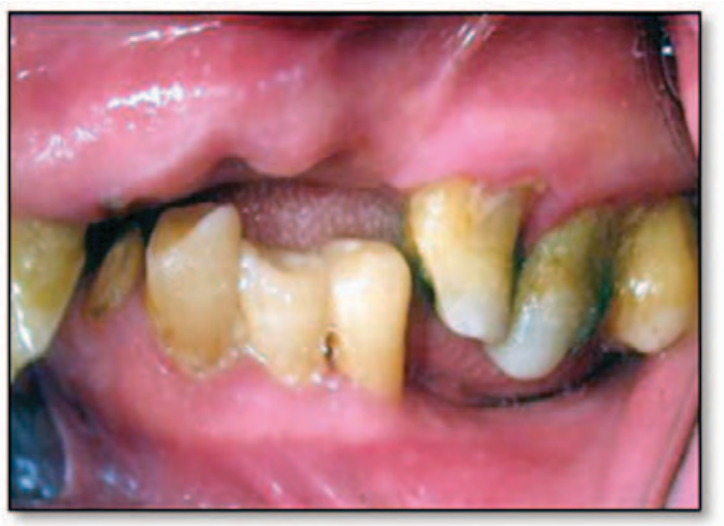

Figure 3. Pretreatment appearance (mirror view of patient's left side). 
The clinical and radiographic examinations revealed furcation involvement of the maxillary left second molar (grade 3) and periapical pathology involving the mandibular central incisors.

\section{Treatment}

It was very apparent that the occlusal plane was very uneven due to supereruption of the remaining posterior teeth. In addition, the crown-root ratio of the mandibular incisors was compromised and greater than a one-to-one relationship, so the mandibular incisors were extracted along with the maxillary right and left maxillary third molars and the left second molar. Panoramic radiograph revealed adequate bone for implant placement (Figure 4).
Diagnostic casts were mounted in centric relation in a semi-adjustable articulator with the aid of an arbitrary facebow and mandibular record base. An occlusal adjustment of the casts and a diagnostic wax-up that included the edentulous segments were performed in the laboratory to achieve the best tooth contour and occlusion at an increased VDO of $3.0 \mathrm{~mm}$, measured at the incisors. The patient's ability to tolerate an increased VDO was evaluated over the course of three months with an RPD.

After evaluation of the patient's comfort and function at the increased VDO, 10 ITI titanium implants (Straumann Dental implants; Institute Straumann AG, Waldenburg, Switzerland) were placed. They included two narrow-neck implants

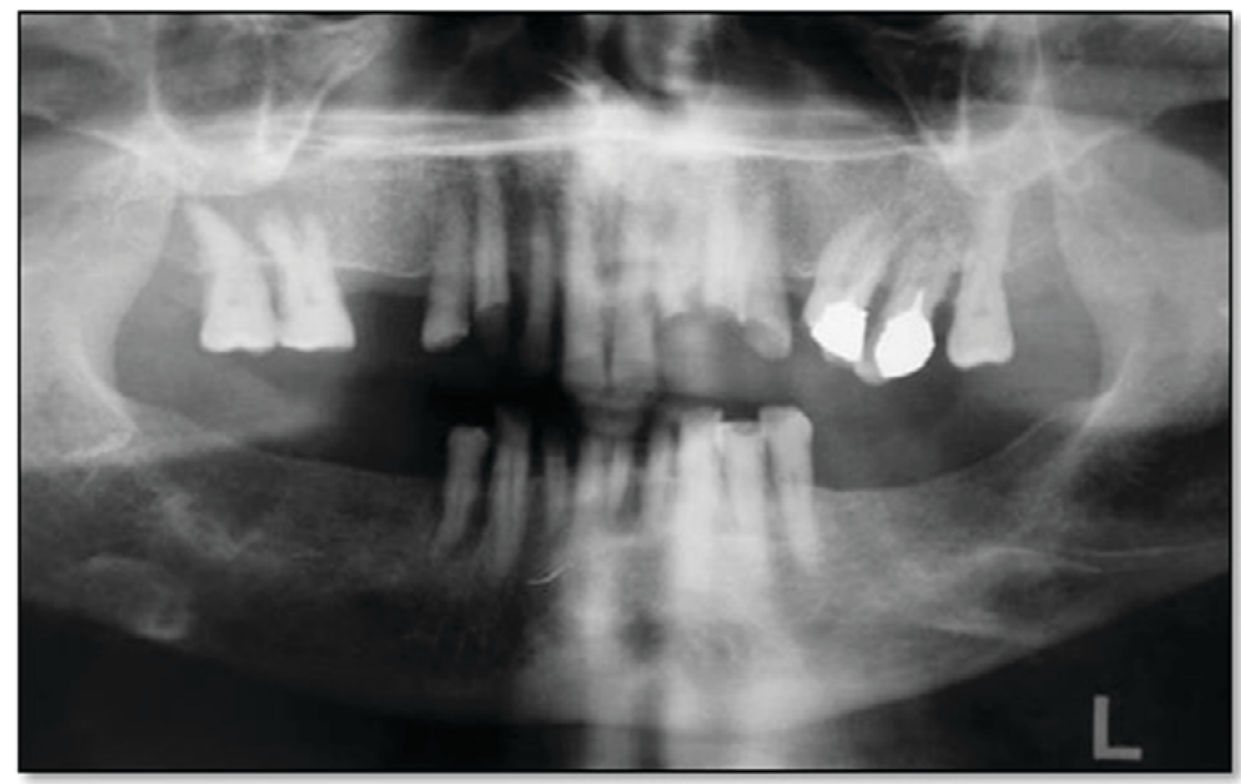

Figure 4. Pretreatment panoramic radiographic.

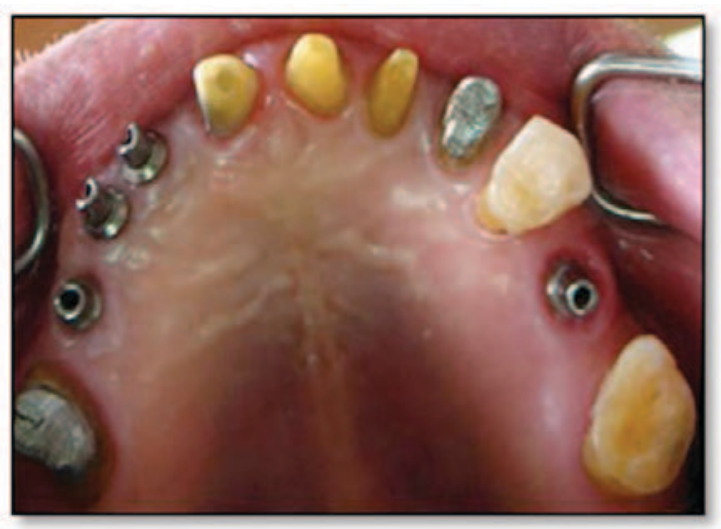

Figure 5. Mirror occlusal view of maxillary prepared teeth and implants.

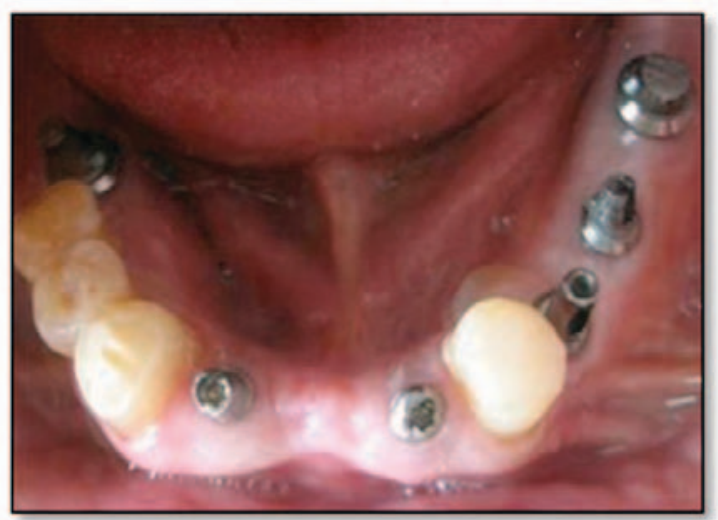

Figure 6. Mirror occlusal view of mandibular implants. 
(NN 3.3×14mm-033.954S) for replacement of mandibular incisors and two wide-neck implants (WN 4.8×10-033.632S) implants for the mandibular first molar area; the remaining implants used were ITI regular-neck implants (RN $4.8 \times 12-033.253 S)$.

During the three-month healing period of the implants, cast post and cores were constructed on the endodontically treated teeth (the maxillary right canine and maxillary left first molar).

The right maxillary incisor had adequate coronal dentin, so only a fiber-reinforced composite post (D.T. Light-Post; RTD, Grenoble, France) was placed. After a satisfactory healing period of three months, implant abutments were selected (Figures 5 and 6).

Next provisional restorations (Tempron; GC Corp., Tokyo, Japan) were fabricated and cemented with zinc oxide cement (Temp Bond NE; Kerr Corp., Orange, CA, USA). The less severely worn mandibular canines were restored conservatively with composite. The anterior guidance was developed on the provisionally restored maxillary anterior teeth. The fixed, implant-retained provisional restorations were comfortable and ensured anterior and posterior stability during the period established to evaluate the patient's tolerance to the newly established VDO (Figure 7).

The provisional restorations were evaluated for esthetic, phonetics, and comfort. Anterior guidance was provided to the technician through the articulator settings and the mounted diagnostic casts of the provisional restorations. ${ }^{?}$

Final impressions were made with vinyl polysiloxane impression materials (President; Coltène/Whaledent AG, Langenau, Germany). Centric occlusion was recorded at the VDO established by the provisional restorations.

Metal-ceramic restorations were fabricated in a nickel-chromium-beryllium base metal alloy (Supercast ${ }^{\mathrm{TM}}$ NP ceramic alloy; Thermabond Alloys Mfg., Los Angeles, CA, USA) and the castings were evaluated intraorally for fit prior to the application of dental porcelain.

The maxillary and mandibular implant-supported restorations were splinted together, as shown in
Figures 10 and 11 . The restorations on natural dentition were cemented with a glass ionomer cement (GC Fuji 1, light yellow, luting cement; GC Corp., Tokyo, Japan). The implant-supported restorations were cemented with a provisional luting agent (Temp-Bond NE; Kerr, Orange, CA, USA) as shown in Figures 8 and 9. After six months, these restorations were cemented with

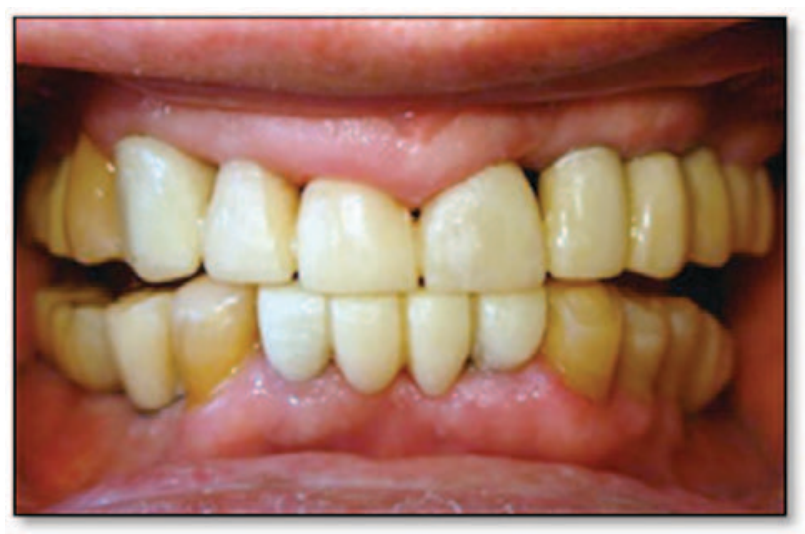

Figure 7. Provisional restorations in the protrusive position.

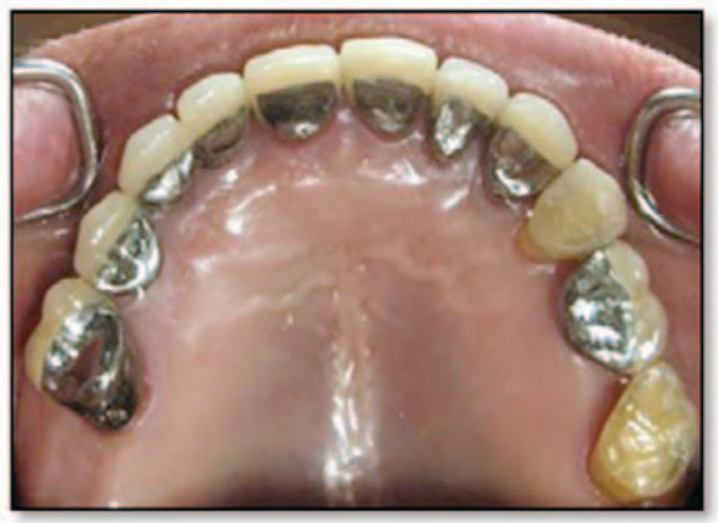

Figure 8. Mirror occlusal view of final maxillary restorations.

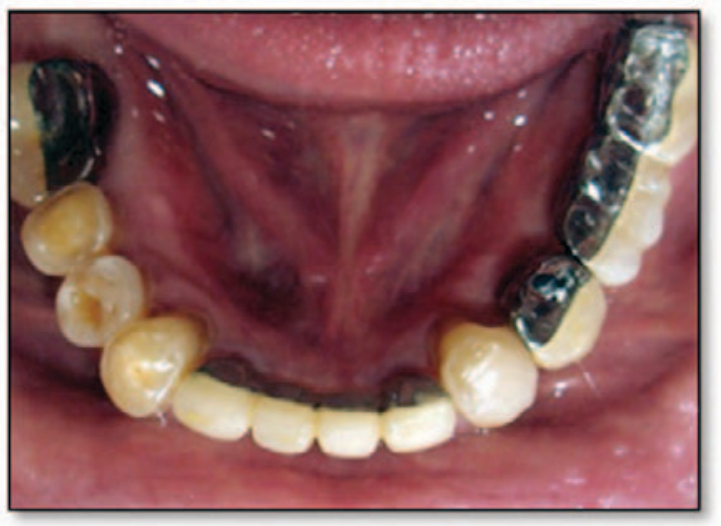

Figure 9. Mirror occlusal view of final mandibular restorations. 
zinc phosphate cement (Richter \& Hoffmann, Berlin, Germany)

The occlusion was developed with maximum intercusal contact in the centric occlusion position with a $3.0 \mathrm{~mm}$ increase in the VDO (Figure 10).

Centric stops on the maxillary incisors were located on metal surfaces of the metal-ceramic crowns. A canine protected occlusion was established to reduce lateral forces on the mandibular implant-supported prosthesis. A hard nightguard was made for the patient to wear at night. The patient was placed on a strict recall schedule for hygiene control and monitoring of the prostheses and the implants.

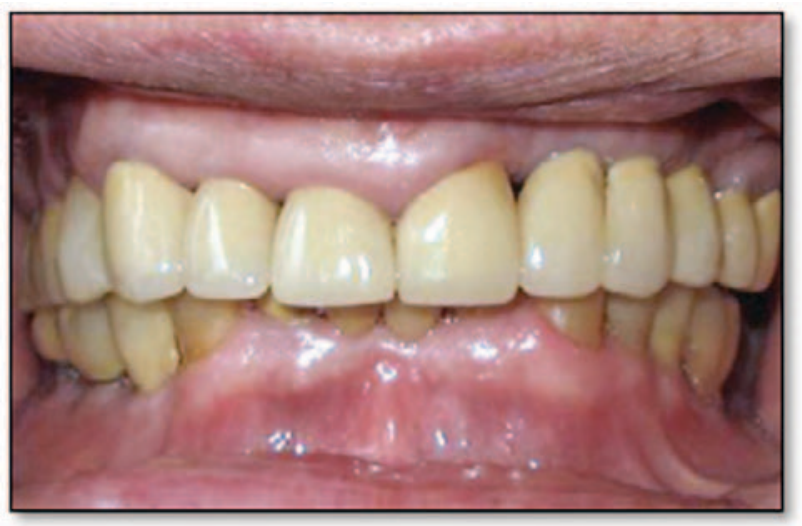

At the one-year follow-up appointment, the patient was functioning well and did not report any problems (Figure 11).

\section{Discussion}

Oral rehabilitation of a patient with severe tooth wear and a long edentulous span can be a great challenge, for both the dentist and the patient.

Complete reconstruction has the benefit of allowing the rehabilitating dentist to restore to ideal occlusion. A simple treatment option available for the worn dentition is the restoration with overlay removable partial dentures. This is a cost-effective treatment option, and with removable partial dentures, the patient can easily perform routine oral maintenance. ${ }^{8-10}$ Patients tend to dislike RPDs because they are bulky and uncomfortable and can become unstable. Several clinical reports described the occlusal rehabilitation of a partially edentulous patient using implants. ${ }^{11,12}$ Yunus et al. ${ }^{11}$ described a successful occlusal rehabilitation with implants of a partially edentulous patient with the loss of posterior support and severely worn dentition. ${ }^{11}$ Balshi and Wolfinger ${ }^{12}$ reported the occlusal rehabilitation of a patient with loss of posterior support and tooth wear.

Figure 10. Final restoration in anterior view.

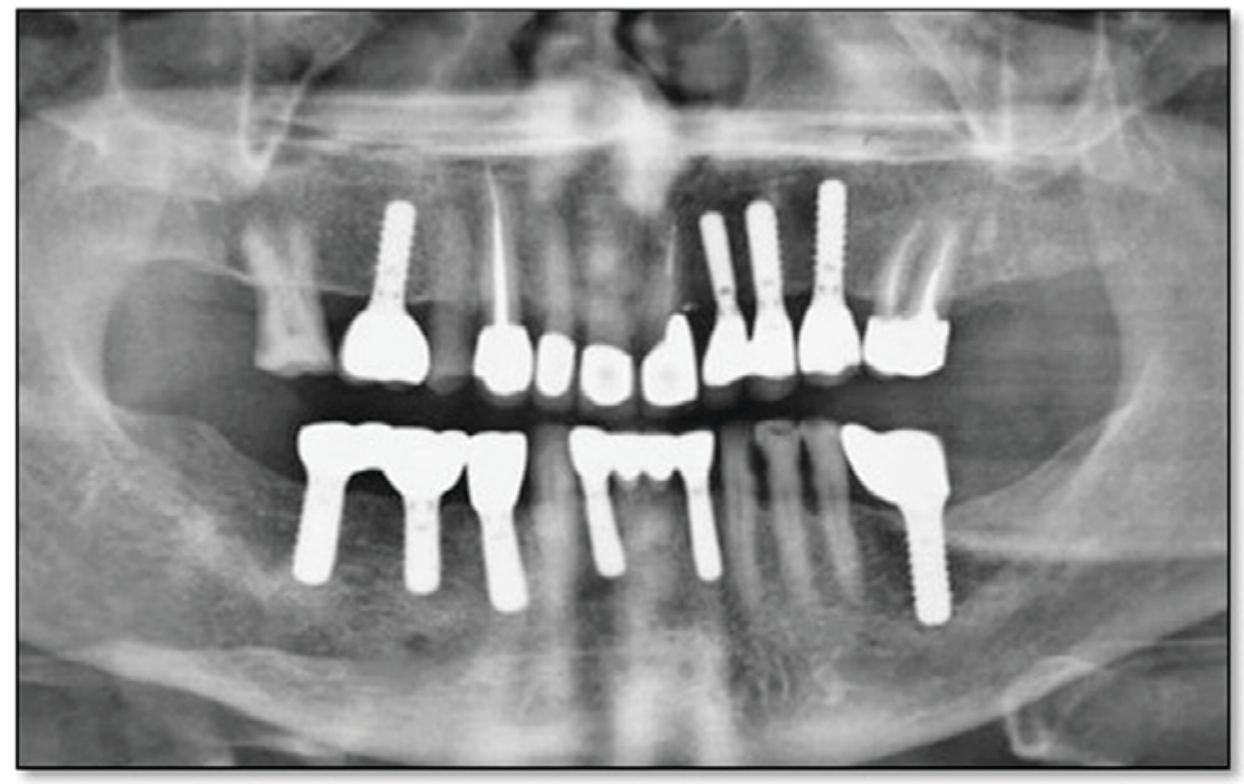

Figure 11. Final radiographic view. 
In the case presented here, an organized approach to occlusal reconstruction with fixed restorations was implemented, and osseointegrated implants were used to support the maxillary and mandibular prostheses. The use of dental implants eliminated the need for removable partial dentures in both the provisional and final restorations. Natural teeth were retained because they had a favorable prognosis and adequate bone existed to permit the placement of dental implants in both the maxilla and the mandible.

In patients with comparable clinical conditions, a similar treatment plan could be considered. However, follow-up appointments for refinements of the occlusion and regular, periodic oral hygiene monitoring are a must. Such patients should always be provided with a hard nightguard and advised to wear it routinely. ${ }^{?}$

\section{Conclusion}

The successful rehabilitation of a patient with severe overbite and supereruption of teeth resulting from the loss of posterior support and severe wear of maxillary and mandibular incisors was described. The treatment plan included occlusal plane correction and the placement of 10 dental implants.

\section{Clinical Significance}

The main indications for implant-supported restorations in a partially edentulous patient are the elimination of RPDs with free-end distal extension bases and the long edentulous span. With the advent of dental implants, the patient can benefit from fixed restorations. However, success with implant-supported prostheses requires the same or more attention to detail and careful treatment planning than conventional fixed prosthodontics.

\section{References}

1. Misch CE. Dental implant prosthetics. St. Louis: Mosby; 2004. p. 1-27.

2. Sheppard IM. Denture base dislodgement during mastication. J Prosthet Dent. 1963; 13:462-8.
3. Jones SD, Jones FR. Tissue-integrated implants for the partially edentulous patient. J Prosthet Dent. 1988; 60(3):349-54.

4. Rosenstiel SF, Land MF, Fujimoto J. Contemporary fixed prosthodontics. 4th ed. St. Louis: Mosby; 2006. P. 381.

5. Nevins M, Langer B. The successful application of osseointegrated implants to the posterior jaw: a long-term retrospective study. Int J Oral Maxillofac Implants. 1993; 8(4): 428-32.

6. Ozkan Y, Ozcan M, Akoglu B, Ucankale M, Kulak-Ozkan Y. Three-year treatment outcomes with three brands of implants placed in the posterior maxilla and mandible of partially edentulous patients. J Prosthet Dent. 2007; 97(2):78-84.

7. Dawson PE. Functional occlusion: from TMJ to smile design. 1st ed. St. Louis: Mosby; 2006. p. 430-52.

8. Windchy AM, Morris JC. An alternative treatment with the overlay removable partial denture: a clinical report. J Prosthet Dent. 1998; 79(3):249-53.

9. Trushkowsky RD, Guiv B. Restoration of occlusal vertical dimension by means of a silica-coated onlay removable partial denture in conjunction with dentin bonding: a clinical report. J Prosthet Dent. 1991; 66(3):283-6.

10. Hemmings KW, Howlett JA, Woodley NJ, Griffiths BM. Partial dentures for patients with advanced tooth wear. Dent Update. 1995; 22(2):52-9.

11. Yunus $\mathrm{N}$, Abdullah $\mathrm{H}$, Hanapiah $\mathrm{F}$. The use of implants in the occlusal rehabilitation of a partially edentulous patient: a clinical report: J Prosthet Dent. 2001; 85(6):540-3.

12. Balshi TJ, Wolfinger GJ. Restoring lost vertical dimension of occlusion using dental implants: a clinical report. Int J Prosthodont. 1996; 9(5):473-8.

\section{About the Authors}

\section{Azam Sadat Madani, DDS, MS}

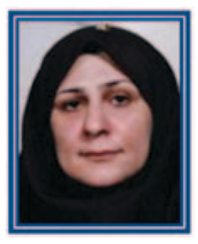

Dr. Madani is an associate professor in the Department of Prosthodontics at the Mashhad Dental School and Dental Research Center of Mashhad University of Medical Sciences, Mashhad, Iran. 
Amir Moeintaghavi, DDS, MS

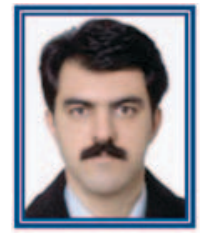

Dr. Moeintaghavi is an associate professor in the Department of Periodontics at the Mashhad Dental School and Dental Research Center of Mashhad University of Medical Sciences, Mashhad, Iran.

Maryam Rezaeei, DDS, MS

(Corresponding Author)

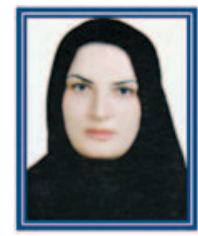

Dr. Rezaeei is an assistant professor in the Department of Prosthodontics at the Babol Dental School and the Dental Research Center of Babol University of Medical Sciences, Babol, Iran.

e-mail: dr.rezaeei@yahoo.com

\section{Acknowledgement}

This study was supported by a grant from the research council of Mashhad University of Medical Sciences, Madhhad, Iran. 